\title{
Aerosol boxes decrease aerosol exposure only in depressurized rooms during aerosol-generating procedures in a simulation study
}

\author{
Wataru Sakai $^{1}$ [D $\cdot$ Gen Hasegawa $^{1} \cdot$ Tomohiro Chaki $^{1} \cdot$ Shunsuke Tachibana $^{1} \cdot$ Michiaki Yamakage $^{1}$
}

Received: 25 February 2021 / Accepted: 30 August 2021 / Published online: 3 September 2021

(c) Japanese Society of Anesthesiologists 2021

\begin{abstract}
Purpose The aim of this study was to compare aerosol exposure with or without an aerosol box in a pressurized/depressurized room during aerosol-generating procedures using an experimental model.

Methods Cake flour (aerosol model) was expelled from an advanced life support training mannequin. The primary outcome measure was the number of $0.3-10 \mu \mathrm{m}$-sized particles at three locations corresponding to the physician, medical staff, and environmental aerosol exposure levels. The aerosol dispersion was visualized using a high-resolution video. The number of expelled particles was measured after artificial coughing during simulated tracheal intubation and extubation in four situations, with or without an aerosol box in a pressurized or depressurized room $(\leq 2.5 \mathrm{~Pa})$.

Results The particles arising from tracheal intubation at the three positions in the four groups differed significantly in size $(p<0.05)$. The sizes of particles arising from extubation at the physicians' and medical staff's faces in the four groups differed significantly in size $(p<0.05)$. Post hoc analysis showed that the counts of all particles at the three positions were significantly lower in the depressurized room with an aerosol box than in the pressurized room without an aerosol box during tracheal intubation $(p<0.05$ at three positions) and extubation $(p<0.05)$ at the physician's and medical staff's positions). Visual assessments supported these results.

Conclusion The aerosol box decreased the exposure of the aerosol to the physician, medical staff, and environment during aerosol-generating procedures in the depressurized room only.
\end{abstract}

Keywords Aerosol dispersion · Visualization assessment $\cdot$ Aerosol box $\cdot$ COVID-19

\section{Introduction}

Prevention of infectious transmission to healthcare providers is a public health priority, which has become especially evident during the SARS-CoV-19 pandemic. Polymerase chain reaction testing and thoracic computed tomography cannot completely diagnose all cases of SARS-CoV-19 infection [1]. Aerosol dispersion should be prevented as much as possible with every patient during aerosol-generating procedures (AGPs), such as tracheal intubation and extubation, as exposure to aerosols can be highly infectious. Further novel or currently unknown infectious diseases that can be

Wataru Sakai

sakaiwataru1128@gmail.com

1 Sapporo Medical University School of Medicine, East 17, South 1, Chuo-ku, Sapporo, Hokkaido 060-8556, Japan transmitted through aerosolized particles are likely to appear in the future. Thus, an effective method to prevent aerosol dispersion during AGPs is required.

At the present stage, we need evidence of the barrier effect of aerosol boxes in AGPs. Aerosol boxes are currently the most well-known aerosol barrier during tracheal intubation. However, the protective effect against aerosol exposure of aerosol boxes during tracheal intubation is controversial [2-4]. Aerosol boxes are also commonly used during tracheal intubation and extubation to prevent aerosol exposure. Generally, tracheal intubation is performed at the sniffing position, but extubation is performed at the neutral head position. In addition, a patients' cough creates aerosols and enhances the flow driving force during extubation, and because of the difference in the direction of aerosol dispersion between tracheal intubation and extubation, the efficacy of an aerosol box in extubation is not clear. Thus, the efficacy of aerosol boxes in extubation is not clear because 
of these differences in the direction of aerosol dispersion between tracheal intubation and extubation.

It is necessary to evaluate the preventive effects of aerosol boxes in various situations. The pattern of aerosol dispersion is affected by environmental conditions, including room airflow, which is different between pressurized and depressurized rooms [5]. The effectiveness of an aerosol box during AGPs, considering environmental factors, has not yet been determined $[6,7]$. In the current preventive strategy, patients that are at risk of infectious transmission are usually managed in a depressurized room; however, in the pandemic period, these patients may be managed in a pressurized or non-pressurized room due to lack of medical resources. Furthermore, no studies have visually or quantitatively evaluated how aerosol flow changes during AGPs under pressurized and depressurized situations.

The present study was conducted to compare the aerosol exposure levels with or without an aerosol box in a pressurized or depressurized room during AGPs, assuming tracheal intubation without a neuromuscular blockade and extubation procedures, by particle counting and our particle visualization method in a cough simulation model.

\section{Materials and methods}

\section{Cough model}

An advanced life support training mannequin (Laerdal Medical, Tokyo, Japan) was used as the lung and airway model. The stomach of the mannequin was detached, and the esophagus of the mannequin was completely closed with vinyl tape to prevent air leakage. Both lungs of the mannequin were massaged simultaneously, as shown in Supplemental Video 1, and the air in the lungs was expelled from the mouth of the mannequin, which was defined as the artificial cough model. The operator was trained to create the same pressure to generate an artificial cough with a particle image velocimetry system (PIV) (Kato Koken, Kanagawa, Japan) and a spirometry system (MICROSPIRO HI-302, NIHON KOHDEN, Tokyo, Japan). We confirmed that the parameters of the simulated cough model were similar to those of a human cough, as in our preliminary study (Supplemental Table E1). The parameters of the artificial and human coughs in the supine position were measured using a PIV (Kato Koken, Kanagawa, Japan) and a spirometry system (MICROSPIRO HI-302, NIHON KOHDEN, Tokyo, Japan) in a preliminary study (Table 1 and Supplemental Video 1).

\section{Tracers}

In this experimental study, cake flour was used as a tracer because it has been reported to effectively simulate human coughs [8]. We measured the particle size of cake flour using two particle counting devices (LA-o950, HORIBA, Ltd., Kyoto, Japan and Model P8-306, Airy Technology Japan Ltd., Tokyo, Japan), which indicated that cake flour contained particles with sizes in the range of $0.3-280 \mu \mathrm{m}$, corresponding to aerosols and droplets. Before each experiment, $2 \mathrm{~g}$ of cake flour was placed in the mouth of the mannequin (Supplemental Video 1).

\section{Particle counting assessment}

Aerosolized particles with diameters of 0.3, 0.5, 1.0, 2.5, 5.0, and $10 \mu \mathrm{m}$ were measured using a particle counting device (Model P8-306, Airy Technology Japan Ltd., Tokyo, Japan). The measurement accuracy of this device is 0.2 particle count/min according to the manufacturer's instructions, which is a small error rate. Particle counting started after the

Table 1 Environmental factors of the simulation and artificial cough patterns

\begin{tabular}{lll}
\hline Environmental factor & & \\
\hline Air change rate $(/ \mathrm{h})$ & & 35 \\
Fresh air change rate $(/ \mathrm{h})$ & $5.1 \times 5.1 \times 3$ \\
Room size $($ length $\times$ width $\times$ height, $\mathrm{m})$ & & $22-27$ \\
Temperature $\left({ }^{\circ} \mathrm{C}\right)$ & & $50-55$ \\
Humidity $(\%)$ & & 0.35 \\
Perpendicular wind speed $(\mathrm{m} / \mathrm{s})$ & $\leq 2.5$ \\
Atmospheric pressure in depressurized room $(\mathrm{Pa})$ & & $>2.5$ \\
Atmospheric pressure in pressurized room $(\mathrm{Pa})$ & Human & Artificial \\
\hline Parameters of human and artificial coughs in the preliminary study & 10 & 10 \\
\hline Maximum speed around the mouth $(\mathrm{m} / \mathrm{s})$ & 0.05 & 0.1 \\
Duration $(\mathrm{s})$ & $<940$ & $410-450$ \\
Volume $(\mathrm{mL})$ & & \\
\hline
\end{tabular}


aerosolized particle level was below the set baseline (number of $2.5 \mu \mathrm{m}$-diameter particles $<1400$ count $/ \mathrm{min}$ ). Particle counting was conducted for $1 \mathrm{~min}$ before and after the artificial cough to calculate the aerosol exposure level, which is taken as the difference between particle counts before and after the artificial cough. The devices were placed at a height of $170 \mathrm{~cm}$ at three locations (Fig. 1E). At the physician's face, the physician intubated the mannequin's trachea to measure the physician's aerosol exposure level, at the medical staff's face while the nurse assists with tracheal intubation (right side of the mannequin) to measure the nurse's aerosol exposure level, and at the foot of the operating bed ( $2 \mathrm{~m}$ caudally from the mannequin's mouth) to measure the environmental aerosol exposure level.

\section{Particle visualization and image capture}

A projector (EB-1985WU, Epson, Nagano, Japan) was connected to a PC to serve as a strong light source. The EB1985WU projects rectangular light at an intensity of 4800 lumens. The color of the PC monitor was set to the brightest green fluorescence (R:148 G:241 B:94), so the projector produced the brightest green light possible, as both human eyes and cameras are most sensitive to green light, according to a professional optics company (Kato Koken, Kanagawa, Japan) (Supplemental Video 1).

The projector was placed $200-250 \mathrm{~cm}$ caudal to the mannequin (Fig. 1A). Green light was directed to a location for the determination of aerosol spreading (Fig. 1B). The scattering of the expelled cake flour, caused by the Tyndall effect, was observed as an aerosol in bright green light.

Four $4 \mathrm{~K}$ resolution video cameras (SONY PXW-Z90, SONY, Tokyo, Japan) were used simultaneously to assess three-dimensional aerosol dispersion. The video recording settings were as follows: gain of $18 \mathrm{~dB}$, color temperature of $3800 \mathrm{~K}$, and f-stop of 4.4. All videos were taken by professional photographers (Support Services Bureau of Photography, Sapporo Medical University of Medicine, Sapporo, Japan). Each video camera was placed at a different location (side and front of the mannequin, side of the physician's face, and at a $45^{\circ}$ angle from the upper caudal side of the medical staff's face).

\section{Experimental settings}

The experiments were conducted in a standard clinical operating room (Fig. 1a-d and Table 1). The medical staff stood on the patient's right side.

Tracheal intubation and extubation were performed using an $8.0 \mathrm{~mm} \mathrm{Hi-Contour} \mathrm{Oral/Nasal} \mathrm{Tracheal} \mathrm{Tube} \mathrm{Cuffed}$ (Covidien, Dublin, OH, USA). The neck of the mannequin during tracheal intubation and extubation was extended $\left(45^{\circ}\right)$ and in the neutral position, respectively. The artificial cough was expelled when the tracheal tube was fixed at $16 \mathrm{~cm}$ (the tip of the tracheal tube just reached the glottis of the mannequin) at the corner of the mouth during tracheal intubation and extubation to decrease the variation in the artificial cough.

\section{Primary outcomes}

The primary outcomes were the aerosolized particle counts at the three points measured for $1 \mathrm{~min}$ immediately after a simulated cough in the four settings during tracheal intubation or extubation (with an aerosol box during tracheal intubation in a depressurized room (DB; $2.5 \mathrm{~Pa}$ or less) or the pressurized room (PB; more than $2.5 \mathrm{~Pa}$ ) and without an aerosol box during tracheal intubation in a depressurized room (DN) or a pressurized room (PN). The definition of a pressurized and depressurized room was based on the Healthcare Engineering Association of Japan Standard (HEAS-02-2013).

\section{Visualization images of the particles}

Particle visualization images (Fig. 1b) and videos during tracheal intubation without paralysis and extubation in the depressurized and pressurized rooms with an aerosol box were recorded. The entire simulation was recorded, including the removal of an aerosol box.

\section{Statistical assessments}

The data were expressed as means [standard deviation (SD)] and medians [interquartile range (IQR)] for continuous and non-continuous variables, respectively, and analyzed using the Shapiro-Wilk test. The data were also examined by analyzing the variance among the four groups or using the Kruskal-Wallis test. If there were statistically significant differences among the four groups, post hoc analysis was conducted using the Tukey-Kramer test or the Steel-Dwass test to detect this difference. Statistical significance was set at $p<0.05$. Statistical analysis was performed using GraphPad Prism 8.0 (GraphPad Software, La Jolla, CA).

\section{Sample size analysis}

According to our preliminary study, a significant difference occurred when the difference $=817$ and $\mathrm{SD}=425$ for the $2.5 \mu \mathrm{m}$-sized particle counts in the DN and DB. The sample size calculation was conducted using the software R (version 3.5.3) with $\alpha=0.05$, and $1-\beta=0.8$, which showed that six samples were required in each group. Considering the experimental variations, $10 \%$ of the estimated sample size was added, and seven samples were taken in each setting. 

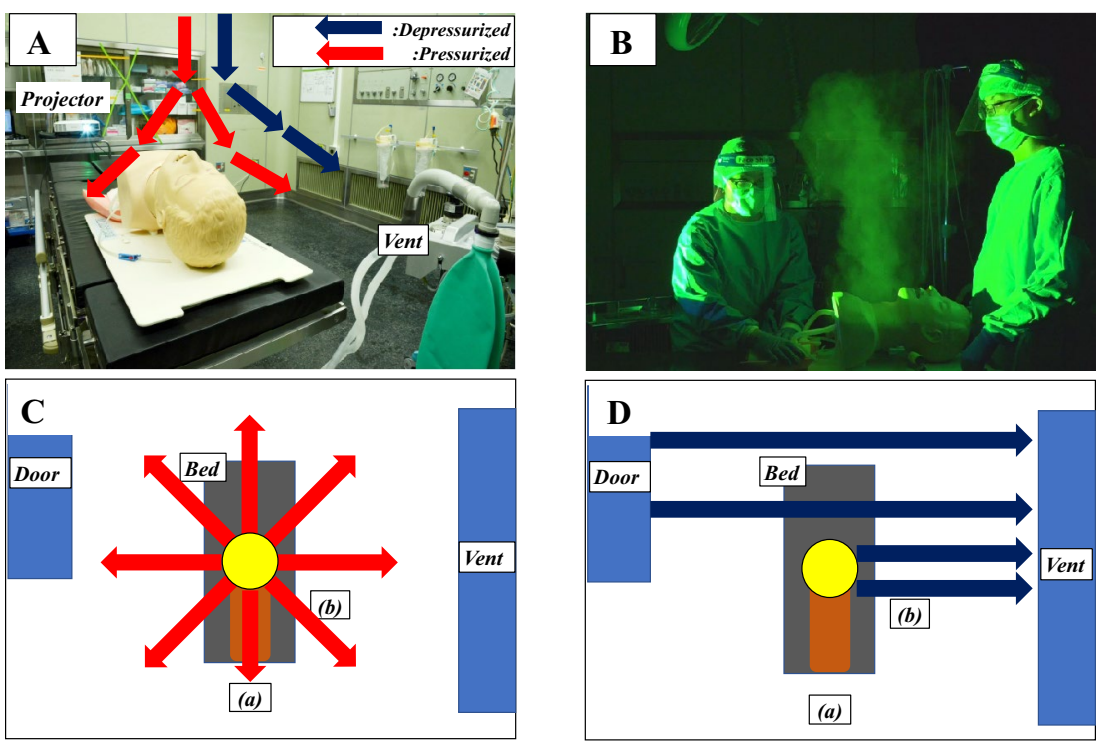

$\mathbf{E}$

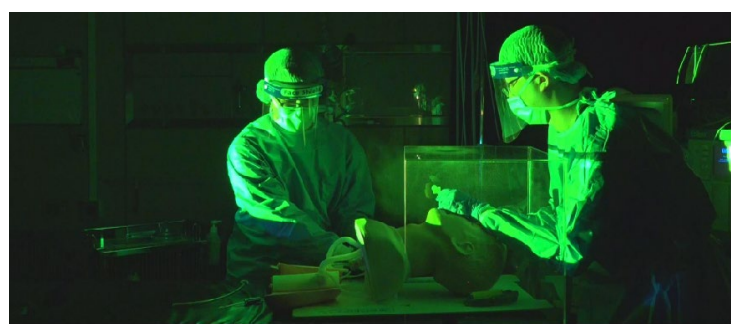

F

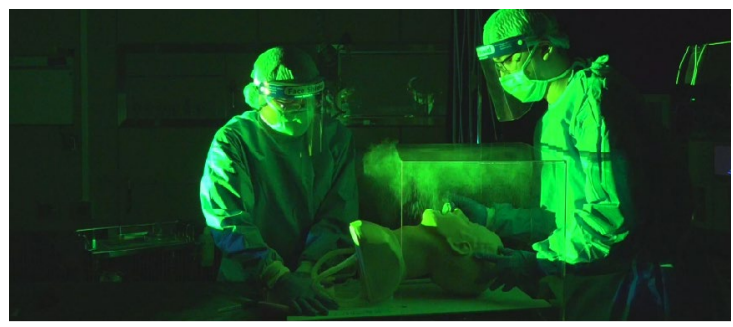

G

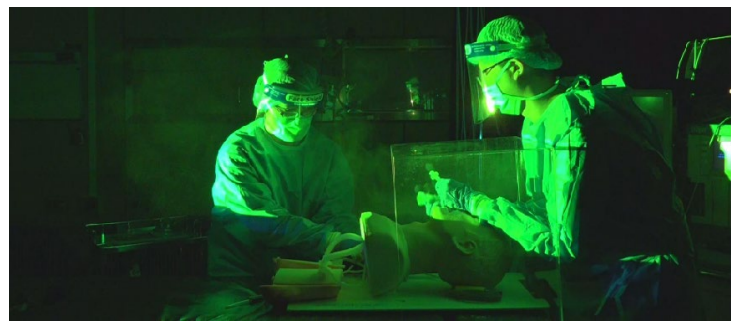

H

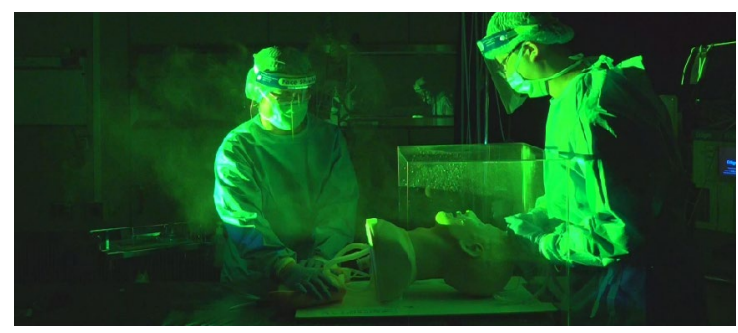


४Fig. 1 Preparation and setup of the experiment to simulate aerosol spreading during tracheal intubation and extubation with an aerosol box in the depressurized and pressurized rooms. The neck position for tracheal intubation and extubation in the depressurized and pressurized rooms can affect the pattern of aerosol spreading. a Preparation and setup of the simulation. The red and blue arrows indicate the wind direction in the depressurized and pressurized rooms, respectively. The vent was operational only in the depressurized room. b The brightest green light was directed to a location where aerosol spreading was determined. c Air direction in the pressurized room. The vent was inoperative in the pressurized room. Red arrow: air flow; yellow circle: air conditioner; brown box: mannequin; a: physician; b: medical staff. d Air direction in the depressurized room. The vent was operational in the depressurized room. Blue arrow: air flow, yellow circle: air conditioner, brown box: mannequin, a: physician, b: medical staff. e Aerosol spread during tracheal intubation without paralysis in the depressurized room. The physician (right) was protected from the expelled aerosolized particles, which dissipated after exiting the aerosol box, but the medical staff (left) was not protected. f Aerosol spread during extubation in the depressurized room. The physician (right) was protected from the expelled aerosolized particles, which dissipated after exiting the aerosol box, but the medical staff (left) was not protected. g Aerosol spread during tracheal intubation without paralysis in the pressurized room. The physician (right) was protected from the expelled aerosolized particles, but the medical staff (left) was highly exposed because the expelled aerosolized particles spread widely. $\mathbf{h}$ Aerosol spread during extubation in the pressurized room. The physician (right) was protected from the expelled aerosolized particles, but the medical staff (left) was highly exposed

\section{Results}

\section{Aerosol exposure levels for the physician, medical staff, and environment}

The sizes of the particles arising from tracheal intubation at all positions in the four groups were significantly different $(p<0.05)$. The sizes of the particles arising from extubation at the physicians' and medical staff's faces in the four groups were significantly different $(p<0.05)$. A summary of the primary outcomes during tracheal intubation and extubation is shown in Figs. 2 and 3 and Supplemental Tables E2 and E3, respectively. In the post hoc analysis, the counts of all different-sized particles from tracheal intubation at the three locations were significantly lower in DB than in PN. The counts of all the different-sized particles from extubation at the physician and medical staff positions were significantly lower in DB than in PN. The counts of all the different-sized particles from tracheal intubation at the medical staff's position were lower in DB than in PB.

\section{Visualization of aerosol dispersion}

The results of the simulations conducted during tracheal intubation and extubation in the depressurized and pressurized rooms are shown in Fig. 1, Supplemental Table E4, and Supplemental Video 1. High-resolution videography detected droplets and aerosolized particles. Two of the whole-view videos showed that the cough vector was to the right and upward, and the cough hit the ceiling of the aerosol box diagonally in all settings. Four videos of tracheal intubation and extubation conducted in the depressurized room showed that the particles dissipated immediately after exiting the aerosol box, whereas, in the pressurized room, the particles remained suspended in air for $15 \mathrm{~s}$ and spread widely around the bed.

\section{i) Aerosol box in the depressurized room}

During tracheal intubation, the side whole-view images showed that the particles spread maximally to $20 \mathrm{~cm}$ caudally and $5 \mathrm{~cm}$ upward upon exiting the aerosol box. The vertical whole-view images showed that the particles spread maximally to $25 \mathrm{~cm}$ sideways and in the direction of the medical staff upon exiting the aerosol box. The images from the nurse's view showed that the particles reached the medical staff's forearm, breast, and face shield. The images from the physician's view showed that the particles moved slowly across the external roof of the aerosol box and reached the physician's face.

During extubation, the side whole-view images showed that the particles spread to a maximum of $15 \mathrm{~cm}$ caudally and $5 \mathrm{~cm}$ upward upon exiting the aerosol box. The vertical whole-view images showed that the particles spread to a maximum of $15 \mathrm{~cm}$ sideways and in the direction of the medical staff upon exiting the aerosol box. The images from the nurse's view showed that the particles reached the medical staff's forearm, breast, and face shield. From the physician's view, the images showed that the particles moved slowly across the external roof of the aerosol box and reached the physician's face.

\section{ii) Aerosol box in a pressurized room}

During tracheal intubation, both the physician and medical staff were exposed to extremely high aerosol and droplet levels.

During extubation, the medical staff was exposed to extremely high aerosol and droplet levels, whereas the physician's exposure level could not be detected. The particles spread widely throughout the room during extubation.

Under all conditions, the aerosol and droplet particles that remained in the aerosol box spread when the box was removed. All the images from the physician's views showed that the physician was exposed to aerosols and droplets as the aerosol box was removed. The end of Supplemental Video 1 focuses on aerosol and droplet dispersion as the box is removed. 
A

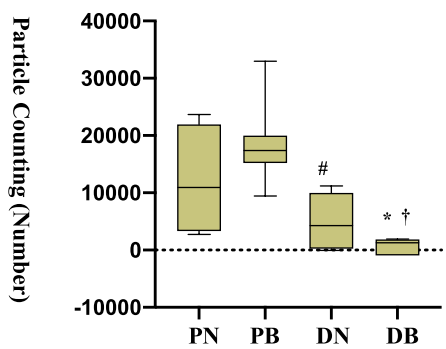

D

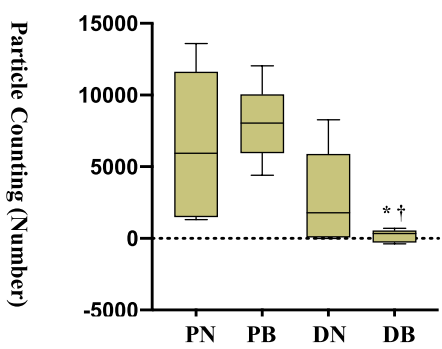

II
B

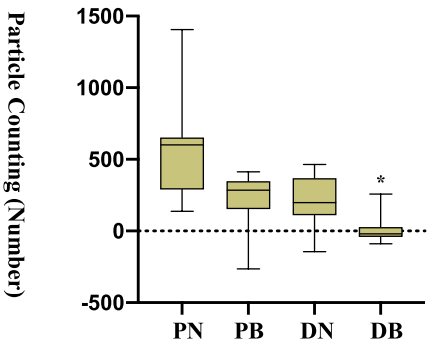

E

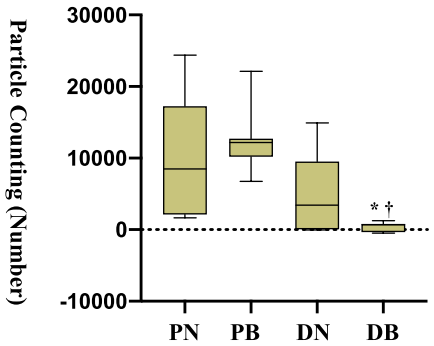

B

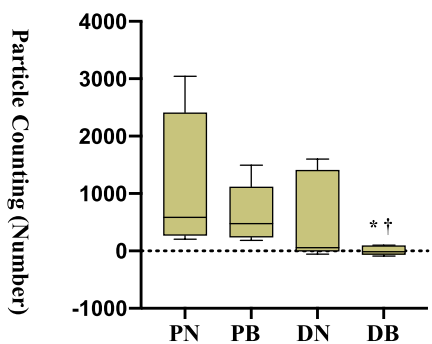

$\mathbf{E}$

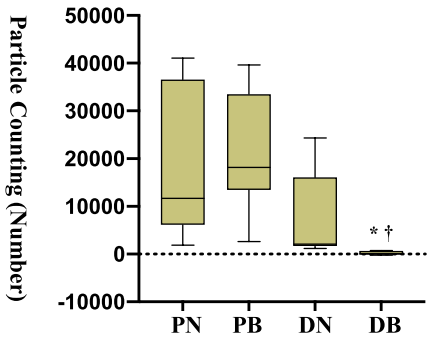

C

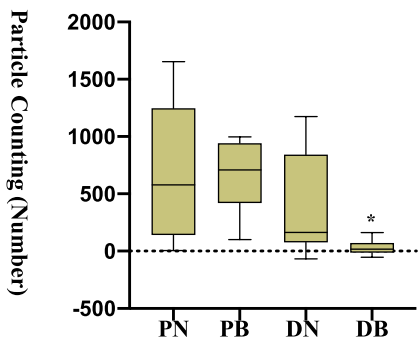

F

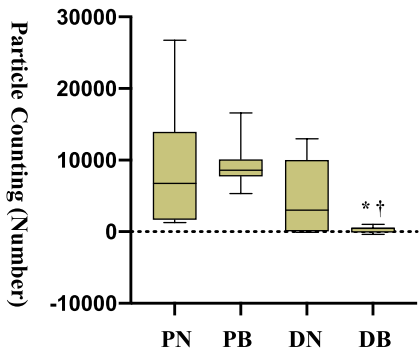

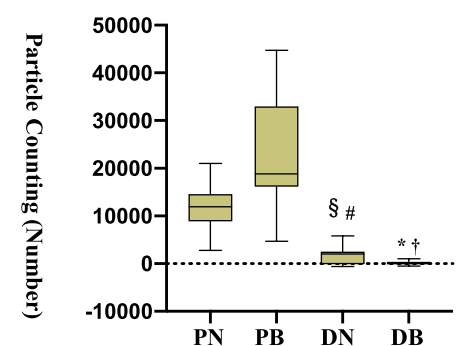

D

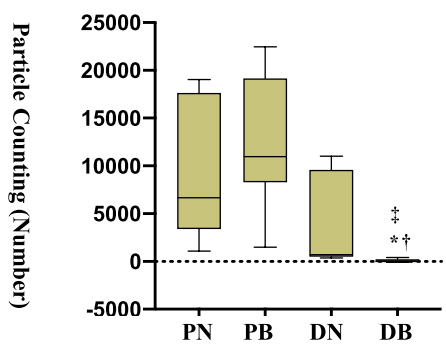

C

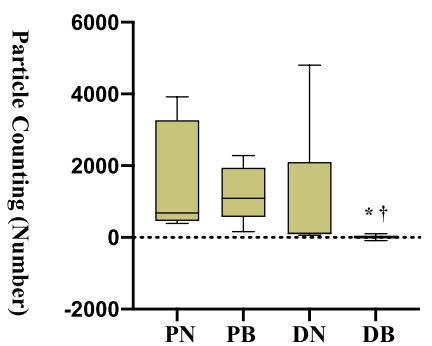

$\mathbf{F}$

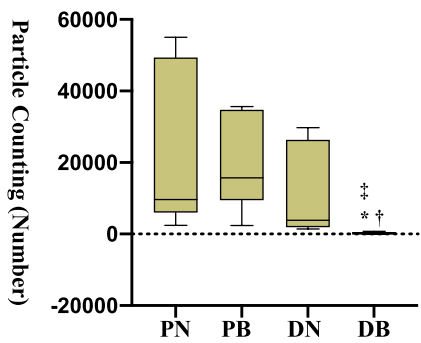

Fig. 2 Summary of particle counts during tracheal intubation. (I) Physician's aerosol exposure level (II) Medical staff's aerosol exposure level (III) Environmental aerosol exposure level. A: $0.3 \mu \mathrm{m}$; B: $0.5 \mu \mathrm{m}$; C: $1.0 \mu \mathrm{m}$; D: $2.5 \mu \mathrm{m}$; E: $5.0 \mu \mathrm{m}$; ${ }^{*}$ significant difference between DB and PN; $\dagger$ significant difference between DB and PB; $\ddagger$ significant difference between $\mathrm{DB}$ and $\mathrm{DN}$; \# significant difference between $\mathrm{PB}$ and DN; $\S$ significant difference between $\mathrm{PN}$ and $\mathrm{DN}$; $\mathrm{PN}$ : in the pressurized room without an aerosol box; PB: in the pressurized room with an aerosol box; $\mathrm{DN}$ : in the depressurized room without an aerosol box; DB: in the depressurized room with an aerosol box 
III

A

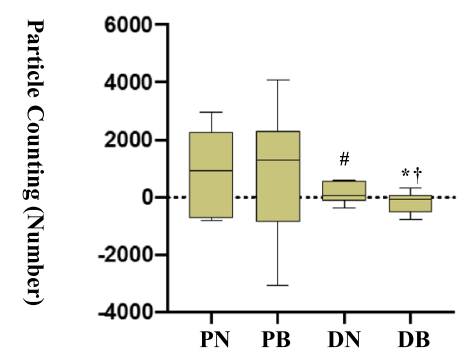

D

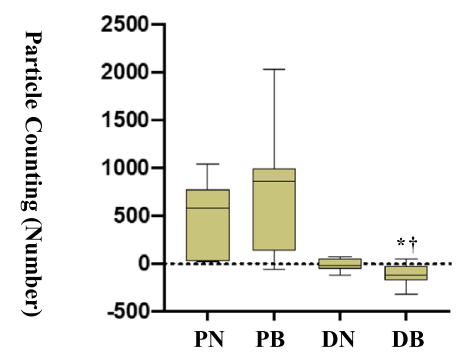

B

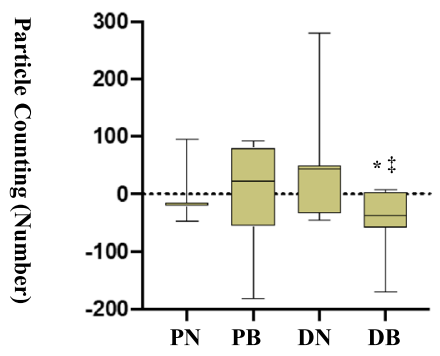

$\mathbf{E}$

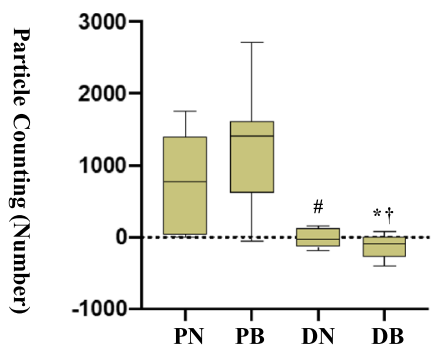

C

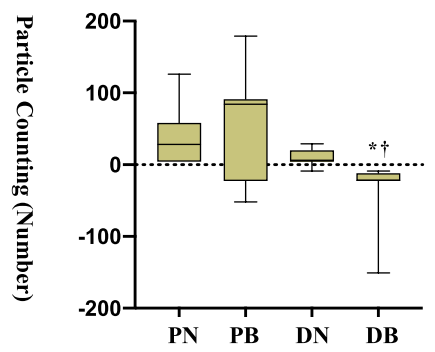

F

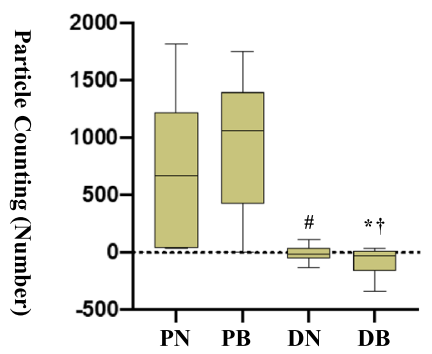

Fig. 2 (continued)

\section{Discussion}

Quantitative, temporal, and visual assessments showed that the aerosol exposure levels of the physician, medical staff, and environment were significantly lower in the depressurized room (with an aerosol box) than in the pressurized room (with and without an aerosol box) during the AGPs. However, the aerosol box in the depressurized room did not completely prevent aerosol dispersion during these procedures. It is of particular importance that the aerosol exposure of the physician was not prevented as the aerosol box was removed in the depressurized and pressurized rooms in all settings.

\section{Difference in aerosol dispersion between pressurized room and depressurized room}

Almost all of the aerosolized particles at the three positions during tracheal intubation and extubation in the depressurized room tended to be smaller than those in the pressurized room with or without an aerosol box. Videography also revealed the difference in aerosol dispersion between pressurized and depressurized rooms. In the depressurized room, aerosols and droplets were immediately eliminated. The main reason for the recommendation that AGPs be performed in a depressurized room rather than in a pressurized room is to limit the spread of viral aerosols outside the room
[9]. However, Qian et al. showed that a depressurized room could not decrease the aerosol exposure [10]. Our depressurized room contained a one-way airflow pathway from the air conditioner to the vent, improving the rate of aerosol and droplet removal [11].

\section{Aerosol dispersal patterns during intubation and extubation}

There were no major differences in the particle counting outcomes between tracheal intubation and extubation because our particle counter could only detect aerosol particles within a size range of $0.3-10 \mu \mathrm{m}$. On the other hand, different aerosol and droplet dispersal patterns between tracheal intubation and extubation could be detected using high-resolution videography. Aerosol and droplet dispersions could spread more caudally in a pressurized room during extubation than during tracheal intubation. Differences in the neck position between tracheal intubation and extubation could lead to different cough vectors and result in variations in spreading patterns.

\section{Efficacy of an aerosol box}

The results did not demonstrate the efficacy of an aerosol box at the three locations in a pressurized room. Only the 
A

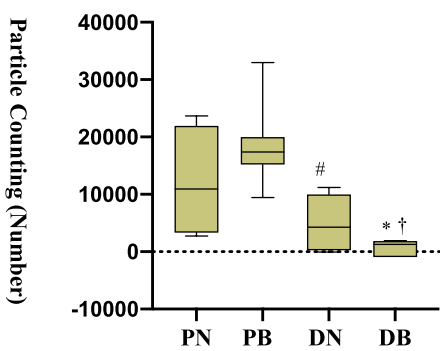

D

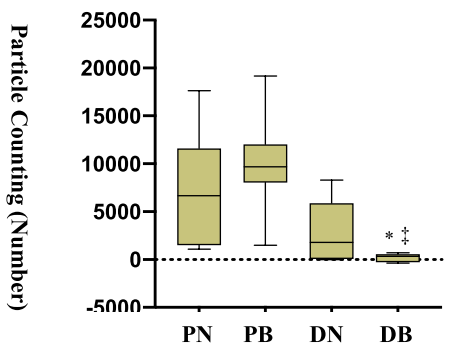

II
B

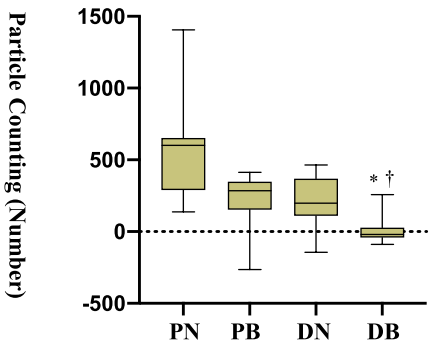

$\mathbf{E}$

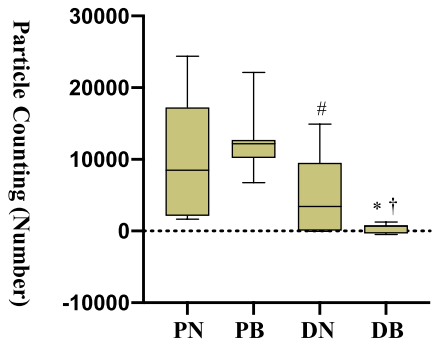

C

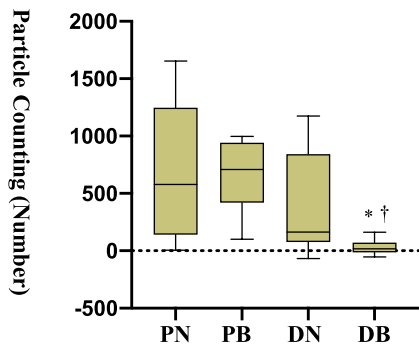

F

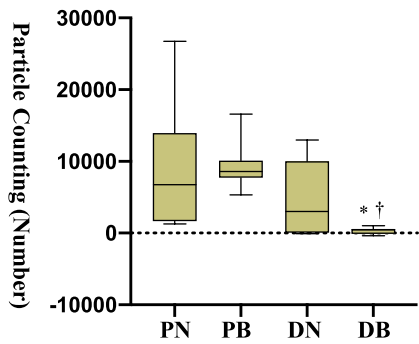

A

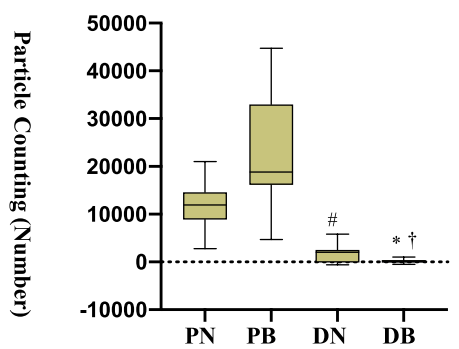

D

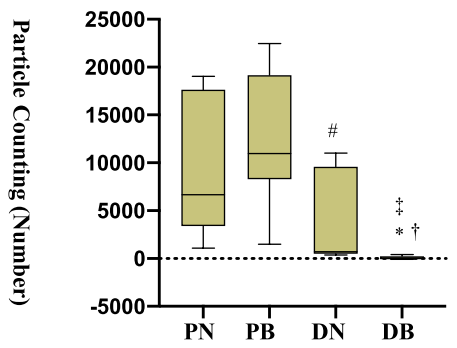

B

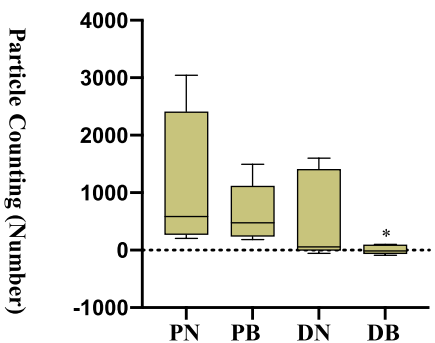

$\mathbf{E}$

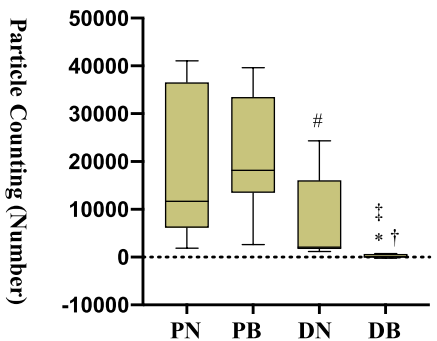

C

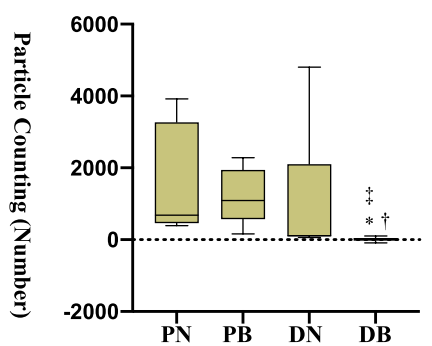

$\mathbf{F}$

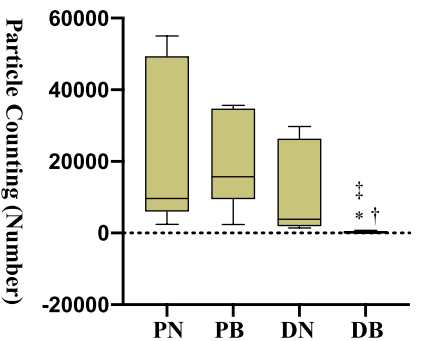

Fig. 3 Summary of particle counts during extubation. (I) Physician's aerosol exposure level (II) Medical staff's aerosol exposure level (III) Environmental aerosol exposure level, A: $0.3 \mu \mathrm{m}, \mathbf{B}: 0.5 \mu \mathrm{m}$; C: $1.0 \mu \mathrm{m}$; D: $2.5 \mu \mathrm{m}$; E: $5.0 \mu \mathrm{m}$; * significant difference between $\mathrm{DB}$ and $\mathrm{PN} ; \dagger$ significant difference between $\mathrm{DB}$ and $\mathrm{PB} ; \ddagger$ signifi- cant difference between DB and DN; \# significant difference between $\mathrm{PB}$ and DN; § significant difference between PN and DN; PN: in the pressurized room without an aerosol box; $\mathrm{PB}$ : in the pressurized room with an aerosol box; DN: in the depressurized room without an aerosol box and DB: in the depressurized room with an aerosol box 


\section{III}

A

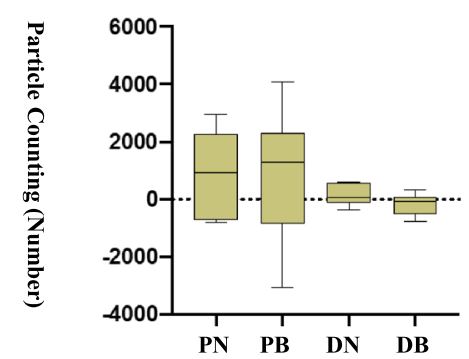

D

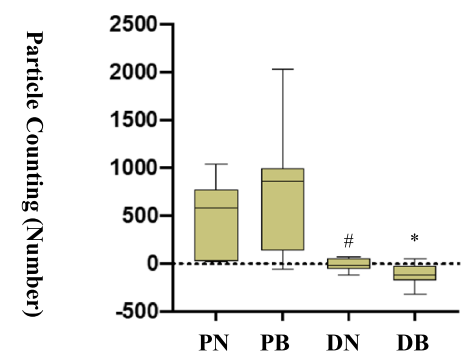

B

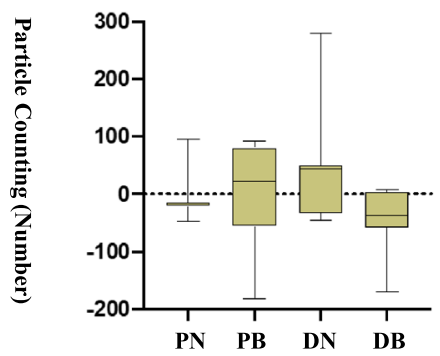

$\mathbf{E}$

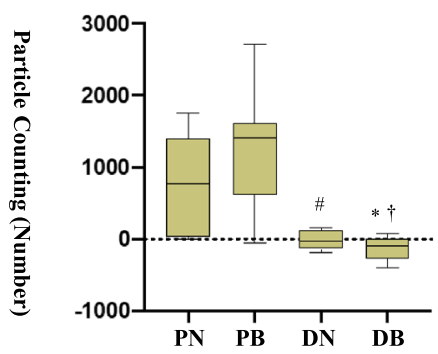

C

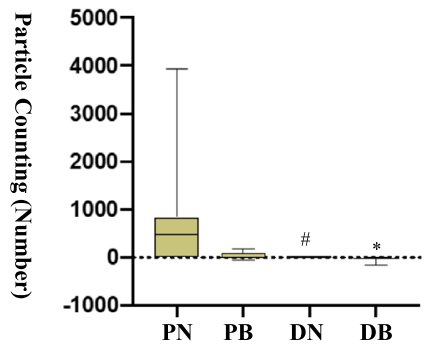

F

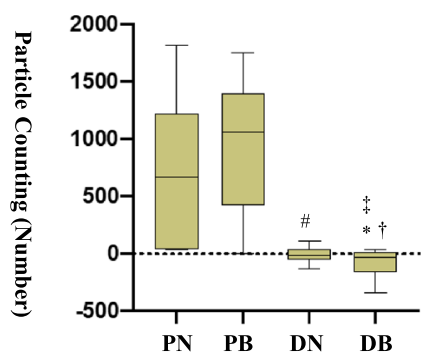

Fig. 3 (continued)

combined use of an aerosol box and a depressurized situation significantly decreased the particle counts at the three locations during tracheal intubation and extubation. However, the quantitative and visual results showed that the aerosol box did not completely prevent aerosol exposure of the physician and medical staff. Even in a depressurized room, the removal of the aerosol box could spread aerosol dispersal, and medical personnel should pay attention to aerosol dispersion until the aerosol box is completely removed after the procedures.

Several studies have shown that an aerosol box can effectively prevent aerosol dispersion [2,3], whereas other studies have not [4]. Azhar et al. showed that an aerosol box significantly reduced the contaminant exposure level but increased tracheal intubation time and reduced operator mobility and visibility in a simulation study [3]. One review article showed that enclosure barriers for airway management should be avoided until adequate evidence is gathered [4]. Several studies have questioned whether an aerosol box can decrease the risk of infection $[6,7]$. Simpson et al. counted aerosolized particles of human coughs in a simulation study and showed that the protective effectiveness of an aerosol box against aerosol exposure was limited [6]. An aerosol box is commonly used as an aerosol barrier during extubation. Unfortunately, in these studies, no protective effects of an aerosol box were observed during extubation.

\section{Environmental factors}

Environmental factors, including air and fresh air change rates, room size, temperature, humidity, airflow and turbulence, atmospheric pressure in depressurized and pressurized rooms, bed position, patient's head position during AGPs, and the position and posture of the physician and medical staff, could affect aerosol dispersion [5, 12-14]. All these factors could differ between countries, hospitals, and rooms. The particle visualization method developed in this study allows clinicians to independently assess aerosol dispersion using common items.

\section{Limitations}

As a limitation, the diameter of the cake flour particles was in the range $0.3-280 \mu \mathrm{m}$, which resulted in a somewhat artificial human cough pattern because of the aerodynamics (Supplemental Video 1) of particles with different diameters, which affected aerosol spread [14]. Because cake flour does not include small, aerosolized particles $(0.01-0.3 \mu \mathrm{m})$ and even high-resolution cameras cannot detect aerosolized 
particles of all sizes, clinical aerosol exposure is likely greater than that determined in the current experiment.

\section{Conclusion}

The results presented herein revealed that an aerosol box during AGPs decreased the aerosol exposure levels of the physician, medical staff, and environment only in the depressurized room, but not in the pressurized room.

Supplementary Information The online version contains supplementary material available at https://doi.org/10.1007/s00540-021-02997-7.

Acknowledgements The authors are grateful to Kato Koken (Kanagawa, Japan) and HORIBA, Ltd. (Kyoto, Japan) for collaborating with this work at no cost.

Author contributions WS: This author served as the global chief investigator for this study, designed the work, acquired for the work, analyzed and interpreted the data, and drafted the work. GH: This author designed the work, helped experiments, interpreted the data and revised the work critically. TC: This author designed the work, helped experiments, acquired for the work, analyzed and interpreted the data for the work, revised the work critically and proofread the draft. ST: This author designed the work, helped experiments, acquired for the work, analyzed and interpreted the data, and revised the work critically. MY: This author designed the work, analyzed and interpreted the data, and revised the work critically. WS, GH, TC, ST and MY approved the final version to be published and agreed to be accountability for all aspects of the work in ensuring that questions related to the accuracy or integrity of any part of the work are appropriately investigated and resolved.

\section{Funding No.}

Data availability All data generated or analyzed during this study are included in this published article and in the supplementary information files.

\section{Declarations}

Conflict of interest All of the equipment, staff, measurement processing, and analyses were carried out using only our department's resources. Kato Koken (Kanagawa, Japan) and HORIBA, Ltd. (Kyoto, Japan) for collaborating with this work at no cost. We declare that all of the authors do not have any conflict of interest.

Ethical approval Not applicable.

Consent to participate Not applicable.

Consent for publication Not applicable.

\section{References}

1. Tang YW, Schmitz JE, Persing DH, Stratton CW. Laboratory diagnosis of COVID-19: current issues and challenges. J Clin Microbiol. 2020. https://doi.org/10.1128/JCM.00512-20.
2. Canelli R, Connor CW, Gonzalez M, Nozari A, Ortega R. Barrier enclosure during endotracheal intubation. N Engl J Med. 2020;382:1957-8.

3. Azhar MN, Bustam A, Poh K, Zahedi AZA, Nazri MZAM, Ariffin MAA, Yusuf MHM, Zambri A, Chong JYO, Kamarudin A, Ang BT, Iskandar A, Chew KS. COVID-19 aerosol box as protection from droplet and aerosol contaminations in healthcare workers performing airway intubation: a randomised cross-over simulation study. Emerg Med J. 2020;38:111-7.

4. Sorbello M, Rosenblatt W, Hofmeyr R, Greif R, Urdaneta F. Aerosol boxes and barrier enclosures for airway management in COVID-19 patients: a scoping review and narrative synthesis. $\mathrm{Br}$ J Anaesth. 2020;125(6):880-94.

5. Elazhary MA, Derbyshire JB. Effect of temperature, relative humidity and medium on the aerosol stability of infectious bovine rhinotracheitis virus. Can J Comp Med. 1979;43:158-67.

6. Simpson JP, Wong DN, Verco L, Carter R, Dzidowski M, Chan PY. Measurement of airborne particle exposure during simulated tracheal intubation using various proposed aerosol containment devices during the COVID-19 pandemic. Anaesthesia. 2020;75:1587-95.

7. Begley JL, Lavery KE, Nickson CP, Brewster DJ. The aerosol box for intubation in coronavirus disease 2019 patients: an in-situ simulation crossover study. Anaesthesia. 2020;75:1014-21.

8. Zhu S, Kato S, Yang JH. Study on transport characteristics of saliva droplets produced by coughing in a calm indoor environment. Building Environ. 2006;41:1691-702.

9. Tsui $\mathrm{BCH}$, Pan S. Are aerosol-generating procedures safer in an airborne infection isolation room or operating room? Br J Anaesth. 2020;125(6):e485-7.

10. Qian H, Li Y. Removal of exhaled particles by ventilation and deposition in a multibed airborne infection isolation room. Indoor Air. 2010;20(4):284-97.

11. Grosskopf K. Air change rate vs airflow pathway: bioaerosol containment and removal in patient rooms. Antimicrob Resist Infect Control. 2015;4:94.

12. Bello A, Quinn MM, Perry MJ, Milton DK. Quantitative assessment of airborne exposures generated during common cleaning tasks: a pilot study. Environ Health. 2010;9:76.

13. Ciofi-Silva CL, Hansen LL, Almeida AG, Kawagoe JY, Padoveze MC, Graziano KU. Negative pressure of the environmental air in the cleaning area of the materials and sterilization center: a systematic review. Rev Lat Am Enfermagem. 2010. https://doi. org/10.1590/1518-8345.1140.2781.

14. Fabriès JF, Choudat D, Wrobel R, Cloutier Y, Dessanges JF, Villette C, Ava JD, Conso F. Computerized equipment for the delivery of inhaled doses of solid particles in specific bronchial challenge. J Aerosol Med. 2000;13:1-10.

Publisher's Note Springer Nature remains neutral with regard to jurisdictional claims in published maps and institutional affiliations. 